\title{
Fragmentation of clusters and recombination induced by intense and ultrashort X-ray laser pulses
}

\author{
N. Tîmneanu ${ }^{a, *}$, B. Iwan ${ }^{a, \dagger}, *$ J. Andreasson ${ }^{a}$, M. Bergh ${ }^{a}, \ddagger$, M. Seibert ${ }^{a}$, C. Bostedt $^{b, c}$, \\ S. Schorb ${ }^{b, c}$, H. Thomas ${ }^{b, d}$, D. Rupp ${ }^{b}$, T. Gorkhover ${ }^{b}$, M. Adolph $^{b}$, T. Möller ${ }^{b}$,

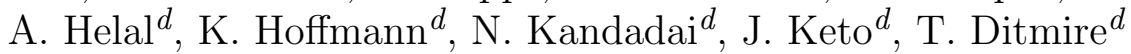 \\ ${ }^{a}$ Laboratory of Molecular Biophysics, Dept. of Cell and Molecular Biology, \\ Uppsala University, Box 596, SE-75124 Uppsala, Sweden \\ ${ }^{b}$ Institut für Optik und Atomare Physik, Technische Universität Berlin, Eugene-Wigner-Bldg. \\ EW 3-1, Hardenbergstrasse 36, D-10623 Berlin, Germany \\ ${ }^{c}$ Linac Coherent Light Source, Stanford Linear Accelerator Center (SLAC) National \\ Accelerator Laboratory, 2575 Sand Hill Road, Menlo Park, CA-94925, USA \\ ${ }^{d}$ Texas Center for High Intensity Laser Science, University of Texas, Austin, TX-78712, USA
}

\begin{abstract}
Understanding the ultrafast dynamics of matter under extreme conditions is relevant for structural studies and plasma physics with X-ray lasers. We used the pulses from free-electron lasers (FLASH in Hamburg and LCLS in Stanford) to trigger X-ray induced explosions in atomic atoms (Xe) and molecular clusters $\left(\mathrm{CH}_{4}\right.$ and $\left.\mathrm{CD}_{4}\right)$. The explosion dynamics depends on cluster size and the intensity of the X-ray pulse, and a transition from Coulomb explosion to hydrodynamic expansion is expected with increasing size and increasing pulse intensity. In methane clusters experiments at FLASH, the time-of-flight spectrometry shows the appearance of molecular adducts which are the result of molecular recombination between ions and molecules. The recombination depends on the cluster size and the expansion mechanism and becomes significant in larger clusters. In Xenon cluster experiments at the LCLS, measurements of the ion charge states in clusters suggest a formation of Xe nanoplasma which expands hydrodynamically. The dominance of low charge states of Xe is due to three-body recombination processes involving electron and Xe ions, and it depends on the X-ray intensity and nanoplasma formation.
\end{abstract}

Keywords: X-ray free electron lasers, atomic and molecular clusters, nanoplasma formation, non-local thermodynamics equilibrium

\section{INTRODUCTION}

X-ray free-electron lasers (XFELs) have opened new and exciting branches of research in all natural sciences, from physics to biology. The Free-electron LASer in Hamburg (FLASH) ${ }^{1}$ in Hamburg and the Linac Coherent Light Source (LCLS) ${ }^{2}$ in Stanford offer an unprecedented insight into the interaction of extremely strong X-ray fields with matter. Very intense and ultra-short X-ray pulses have been used to study ultrafast dynamics in a variety of samples, ranging from free atoms, ${ }^{3}$ atomic clusters, ${ }^{4-7}$ solid targets, ${ }^{8-10}$ and biological structures. ${ }^{11-14}$

Atomic and molecular clusters are very versatile samples. Their size can be adjusted to bridge the gap between molecular and bulk matter, and their atomic composition can be kept simple. Moreover, ionization pathways can be determined in these systems as there is no hidden energy dissipation into the surrounding media. This makes them ideal targets for investigations of ionization and ion acceleration dynamics when the sample reacts to intense and short laser pulses. Understanding the ultrafast dynamics at high fields has important consequences for the field of coherent diffractive imaging, ${ }^{15}$ as well as plasma physics and fusion. ${ }^{16,17}$

* Send correspondence to N. Tîmneanu: nicusor@xray.bmc.uu.se; B. Iwan : bianca@xray.bmc.uu.se $\dagger$ Present address: CEA-Saclay, IRAMIS, Service des Photons, Atomes et Molécules, 91191 Gif-surYvette, France $\ddagger$ Present address: Swedish Defence Research Agency, SE-16490, Stockholm, Sweden

Damage to VUV, EUV, and X-ray Optics IV; and EUV and X-ray Optics: Synergy between Laboratory and Space III, edited by René Hudec, Libor Juha, Saša Bajt, Ladislav Pina, Richard London, Proc. of SPIE Vol. 8777, 87770J (C) 2013 SPIE $\cdot$ CCC code: $0277-786$ X/13/\$18 · doi: 10.1117/12.2019299 
The cluster response to an intense X-ray field depends on the wavelength and intensity of the field, and on the cluster size. For a fixed wavelength, the electrons ejected from atoms in photoionization process can escape the sample or propagate through it and cause further ionizations, in a process dependent on the electron energy and the size of the sample. ${ }^{18}$ As the positive charge of the sample increases at later stages of the exposure, even the fast electrons can be trapped due to the increased positive potential. ${ }^{19}$ At small sample sizes, the explosion is dominated by the repulsion of positive ions left behind by the fast electrons. If most of the electrons are trapped, due to a larger sample size or longer photon wavelength, then the Coulomb interaction is partially screened and the explosion is slowed down. As a net neutral core is formed, the expansion is driven by thermal processes due to the growing electron pressure ${ }^{20,21}$ and the sample is destroyed from the outside toward the center. ${ }^{22,23}$ Larger samples are confined for longer than smaller samples. A transition from Coulomb explosion to a hydrodynamic expansion is expected with increasing sample size. ${ }^{24}$

We have performed several experiments at FLASH and LCLS to characterize the fragmentation of clusters with varying atomic composition, cluster size, laser wavelength, and pulse intensity. At FLASH, we have used intense soft X-rays (92 eV energy) to trigger the explosions of $\mathrm{CH}_{4}$ and $\mathrm{CD}_{4}$ clusters. ${ }^{25}$ Our results showed that the explosion dynamics depends on the clusters size and indicate a transition from Coulomb explosion to a hydrodynamic expansion with increasing cluster size. At LCLS, we have have irradiated Xenon clusters with femtosecond X-rays $\left(850 \mathrm{eV}\right.$ energy). ${ }^{26}$ The results show the formation of Xenon nanoplasma in the intense $\mathrm{X}$-ray pulses, which expands through hydrodynamic expansion. In both cases, the sample fragmentation is accompanied by recombination processes which provide a handle on the understanding of plasma formation and expansion. These results on recombination and the insight on cluster fragmentation are reviewed below in more detail, with emphasis on the plasma dynamics and recombination processes.

\section{EXPERIMENTS}

The experiments on molecular clusters were performed at the BL2 beamline at FLASH. Clusters of methane $\left(\mathrm{CH}_{4}\right)$ and fully deuterated methane $\left(\mathrm{CD}_{4}\right)$ were produced by supersonic expansion of gas through a conical nozzle and were then transported into the experimental interaction region where they were intersected by 15 fs long soft X-ray laser pulses. The laser wavelength was fixed to $13.5 \mathrm{~nm}$ wavelength which corresponds to a photon energy of $92 \mathrm{eV}$. The focal spot diameter was measured to be $20 \mu \mathrm{m}$ at FWHM, and the pulse energy ranging from 10-70 $\mu \mathrm{J}$. The intensity of the laser is variable from shot-to-shot due to the stochastic nature of the lasing process. These give an effective pulse intensity in the range of $10^{13}-10^{15} \mathrm{~W} / \mathrm{cm}^{2}$ with a mean of $5 \times 10^{14}$ $\mathrm{W} / \mathrm{cm}^{2}$ (given a beamline transmission of about $64 \%$ ). ${ }^{27}$

Depending on the temperature $T$ and pressure $p$ and the geometry of the source nozzle (the effective diameter is denoted $d$ ) during cluster production, the mean number of molecules in a cluster $\langle N\rangle$ can be estimated by employing Hagena's empirical formula. ${ }^{28,29}$

$$
\Gamma=\frac{K p d^{0.85}}{T^{2.2875}}, \quad\langle N\rangle= \begin{cases}38.4\left(\frac{\Gamma}{1000}\right)^{1.64} & 350<\Gamma<1800, \\ 33\left(\frac{\Gamma}{1000}\right)^{2.35} & 1800<\Gamma\end{cases}
$$

$\Gamma$ is the Hagena parameter and $K$ is a gas specific sublimation constant. For methane, this is taken to be 2360. Variations in the temperature of the nozzle $(160 \mathrm{~K}<T<200 \mathrm{~K})$ and in the gas pressure $(1.8$ bar $<p<$ 18 bar) can be used to control the cluster size. The obtained clusters could be varied in size from 1,000 molecule up to 250,000 molecules per cluster. These sizes correspond to radii between $2.46 \mathrm{~nm}$ to $15.5 \mathrm{~nm}$, considering spherical clusters at liquid methane density.

The experimental interaction region is located between the plates of a time of flight (TOF) detector. Clusters illuminated by the X-ray pulses will disintegrate into ionized fragments due to the high energy deposition of the soft X-ray pulses. Positively charged ions are then accelerated by the positively charged repeller plate towards a negatively charged multi-channel plate (MCP) detector located at the end of the TOF's field-free drift tube (Figure 1). They impact onto the MCP and their arrival times are recorded resulting in typical time of flight spectra.

To study the effects of the size dependence in the methane clusters, we have analyzed the spectra produced by the X-ray laser with an energy of $43 \pm 1 \mu \mathrm{J}$, corresponding to an intensity on the sample of $5 \times 10^{14} \mathrm{~W} / \mathrm{cm}^{2}$. All spectra in this intensity bin have been averaged. 


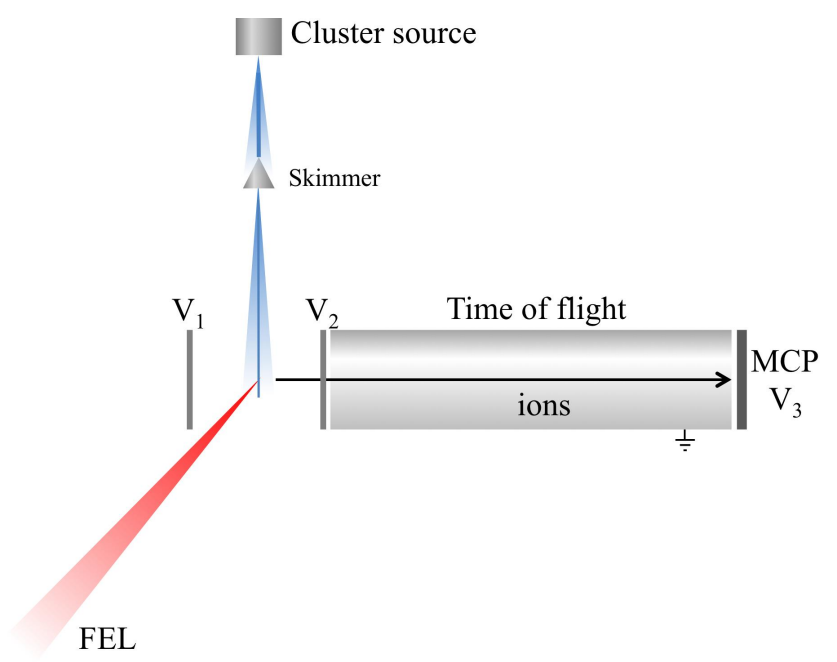

Figure 1. Clusters are produced through supersonic expansion of gas and travel to the interaction region located between the plates of an ion time of flight (TOF) spectrometer. After passing a skimmer which aims to reduce the background, the clusters are exposed to femtosecond X-ray pulses (92 eV at FLASH and $850 \mathrm{eV}$ at LCLS). With given voltages on the TOF plates $\left(\mathrm{V}_{1}, \mathrm{~V}_{2}\right)$, positively charged ions are accelerated towards the field-free drift tube of the TOF spectrometer and are detected by a multi-channel plate (MCP) detector.

The experiments on atomic clusters were conducted at the Atomic and Molecular Optical beamline (AMO) at the LCLS. Clusters of xenon were produced similar to above, through a mechanism of super-sonic expansion of Xe gas through a conical nozzle with $100 \mu \mathrm{m}$ diameter and a $15^{\circ}$ cone angle. A gas pressure of 8.2 bars at 300 $\mathrm{K}$ was expanded through the nozzle, and produced clusters with a statistical size distribution around the mean cluster size of $\langle N\rangle=11,000$ atoms (approximately $5.5 \mathrm{~nm}$ radius). In the formula above, the $\mathrm{K}$ parameter for Xe is taken to be 5550 .

We studied explosions of the Xenon clusters with X-ray laser pulses of $850 \mathrm{eV}$ photons (wavelength $1.45 \mathrm{~nm}$ ) and with a pulse length of $150 \mathrm{fs}$. The X-rays were focused to a spot of $2 \mu \mathrm{m}^{2}$ area and had an average energy of $1.1 \mathrm{~mJ}$ per laser pulse, with a maximum of $2 \mathrm{~mJ}$. Using gas attenuators, we have controlled the intensity in the interaction region over a range from $4 \times 10^{14} \mathrm{~W} / \mathrm{cm}^{2}$ up to $6 \times 10^{16} \mathrm{~W} / \mathrm{cm}^{2}$.

To study the ions generated by the interaction of the X-ray pulses and the clusters, we employed a McLarentype of TOF spectrometer and an MCP detector, similar to the one described above. The obtained spectra have been averaged over several thousands of single-shot LCLS pulses, thereby effectively integrating over the cluster sizes in the statistical distribution and, at fixed laser intensity, the range of intensities on the sample resulting from the shape of the focus (assumed to be Gaussian).

\section{RESULTS}

A qualitative analysis of the mass spectra from the methane experiment at FLASH (Figure 2) reveals significant features that show a size dependency in the fragmentation of the clusters. Shown in the figure are several spectra of molecular and clustered methane molecules, selected for an average intensity of $5 \times 10^{14} \mathrm{~W} / \mathrm{cm}^{2}$. The molecular spectrum exhibits a weak hydrogen $\left(\mathrm{H}^{+}\right)$signal at $\mathrm{m} / \mathrm{q}=1$ and includes peaks of single methane fragments $\left(\mathrm{CH}_{n}^{+}\right)$, with no signs of higher molecular adducts. The insert shows a zoom into the $\mathrm{m} / \mathrm{q}$ region between 12 and 18, with an atomic signal for singly charged carbon $\mathrm{C}^{+}$as well as peaks for $\mathrm{CH}_{n}^{+}$with $n$ ranging from 1 to 4 . A small signal from background water in the experimental chamber can be seen at $n=17-18$, which is also seen when the nozzle source is closed. There are no other signs of ions or fragments with higher $\mathrm{m} / \mathrm{q}$. The spectrum for a cluster size of $\langle N\rangle=20,000$ molecules shows an increase in the overall ion yield, which keeps growing with larger clusters, as seen for a cluster size of $\langle N\rangle=100,000$ molecules. With increasing cluster size, the spectra show a further increase in the yield of single ion species $\mathrm{H}^{+}$and $\mathrm{C}^{+}$. 


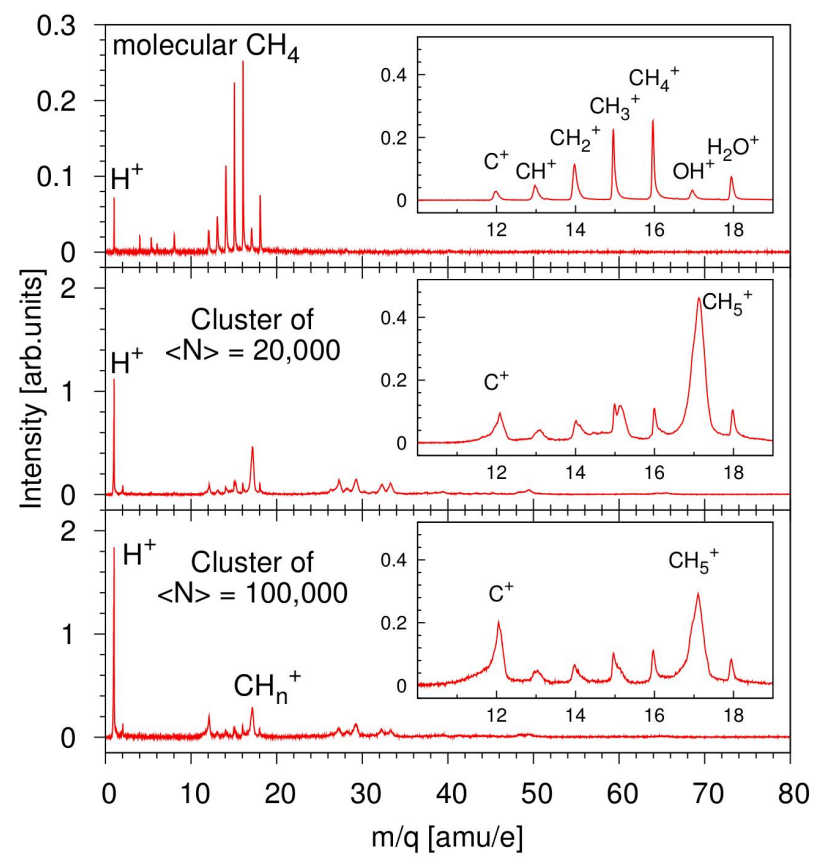

Figure 2. Mass over charge spectra from molecular methane and methane clusters of different sizes irradiated by a 15 fs long FLASH pulse, energy $92 \mathrm{eV}$, with a fixed intensity of $5 \times 10^{14} \mathrm{~W} / \mathrm{cm}^{2}$. From top to bottom, the spectra for $\mathrm{CH}_{4}$ molecules, and average clusters sizes of $\langle N\rangle=20,000$ molecules and $\langle N\rangle=100,000$ molecules. The inset shows a zoom into the region corresponding to $\mathrm{CH}_{n}^{+}$adducts from 12 to 18 . The spectra show an overall increase of $\mathrm{H}^{+}$and $\mathrm{C}^{+}$ions and the emergence of the $\mathrm{CH}_{5}^{+}$and higher adducts in the region $\mathrm{C}_{2} \mathrm{H}_{n}^{+}$in the cluster spectra, which reach a maximum yield at $\langle N\rangle=20,000$. Figure adapted from. ${ }^{25}$

The most prominent feature of the methane spectra is the observation of the protonated methane $\mathrm{CH}_{5}^{+30}$ and other additional adducts at higher $\mathrm{m} / \mathrm{q}$ values $\mathrm{C}_{2} \mathrm{H}_{n}^{+}, n=3 . .9$. These are only observed in the spectra from clusters and give the first indication of molecular recombination during cluster fragmentation. The signal from these fragments grows with increasing cluster size from 1,000 to 20,000 molecules. At large cluster sizes, the yield from these fragments decreases (100,000 shown in Figure 2) and provide the first indication that molecular recombination is a size-dependent effect and that different expansion mechanisms could dominate at different cluster sizes (Coulomb explosion vs. hydrodynamic expansion).

The same observations were made in the experiments on deuterated methane $\mathrm{CD}_{4},{ }^{25}$ which exhibit the same overall increase of the total ion yield and the $\mathrm{D}^{+}$and $\mathrm{C}^{+}$ions with increasing cluster size. We have observed the appearance of similar molecular adducts $\mathrm{CD}_{5}^{+}$and $\mathrm{C}_{2} \mathrm{D}_{n}^{+}$, which show the same behavior with increasing cluster size (yield reaches a maximum for cluster sizes of 20,000 molecules).

We have simulated the interaction of methane clusters with the FLASH pulse using a non - local thermodynamic equilibrium plasma code called CRETIN. ${ }^{31,32}$ This code follows the ion population dynamics and plasma dynamics during and after the pulse, and takes into account radiation transfer, thermal conduction, and electron impact ionization. It also includes electron-ion thermalization and electron-ion recombination. The simulations show that after thermalization, the electron temperature reaches $4 \mathrm{eV}$ and there is an average ionization of 0.5 per carbon atom, for FLASH parameters $5 \times 10^{14} \mathrm{~W} / \mathrm{cm}^{2}$, 15 fs pulses, $92 \mathrm{eV}$ photons. The plasma environment is confined for a longer time with increasing sample size. Simulations on the hydrodynamic expansion of such plasma show a burning rate of $0.01 \mathrm{~nm} / \mathrm{fs}$, a rate which is consistent with the previous experimental observations at FLASH on nanosized polystyrene spheres. ${ }^{33}$ A simulation of the expansion dynamics is shown in Figure 3, which follows the density of the plasma as it expands. The interior of the sample is kept together for a longer 


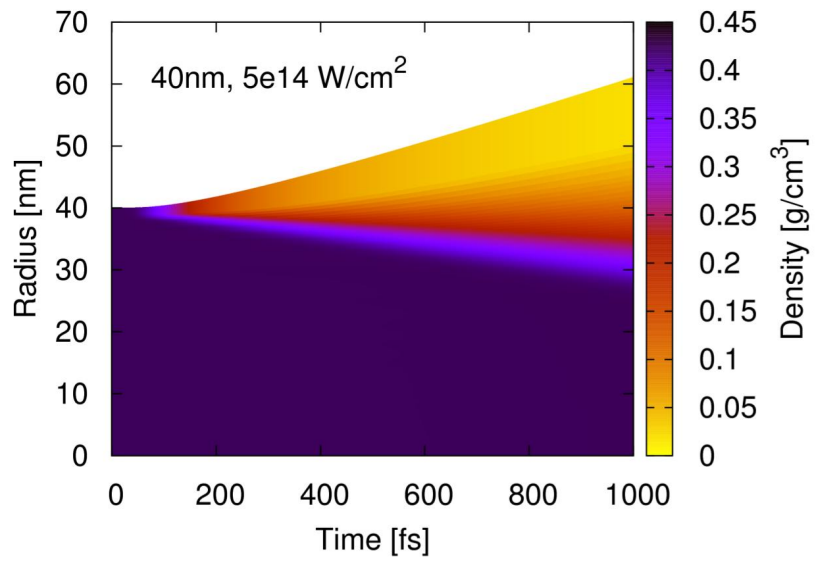

Figure 3. Simulation of a hydrodynamic expansion in a large methane cluster using a non - local thermodynamic equilibrium plasma code, CRETIN. The experimental parameters at FLASH are used: 15 fs soft X-ray pulses, 92 eV energy, $5 \times 10^{14} \mathrm{~W} / \mathrm{cm}^{2}$ intensity. The density of the cluster is shown during a $1 \mathrm{ps}$ simulation and the outer layers of the cluster burn off in a pressure-driven expansion and the sample reduces its size by $10 \mathrm{~nm}$ in 1 ps. This indicates that large clusters will take longer to expand and additional recombination processes can take place.

time, and this allows additional process to take place on longer time scales.

Although CRETin cannot account for bond formation and molecular recombinations which take place on longer time scales, we propose that the formation of the molecular adducts $\mathrm{CH}_{5}^{+}$and $\mathrm{C}_{2} \mathrm{H}_{n}^{+}$proceeds through molecular recombination between the ions and molecules trapped in the core. The decrease in the yield of such adducts with increasing cluster size is connected to the lifetime of the cluster, which is longer for larger clusters. This increases the probability for collisional processes to take place and more decay channels, like dissociation or neutralization, will become accessible.

The experimental results from the xenon experiments at the LCLS (Figure 4) show similar features of recombination process in a plasma environment compared to the FLASH experiment. The atomic spectrum displayed at the top shows the ion charge-state spectra for Xenon atoms irradiated with a high intensity laser $5.6 \times 10^{16}$ $\mathrm{W} / \mathrm{cm}^{2}, 850 \mathrm{eV}$ photon energy and 150 long pulses. High charge states of xenon up to $\mathrm{Xe}^{+26}$ dominate the atomic spectrum, while low charge states are almost completely absent. This feature is easily explained by the sequential ionization of the Xe atoms due to the high x-ray fluence, which can ionize the Xe ions all the way up to $\mathrm{Xe}^{+26}$ (reaching $\mathrm{Xe}^{+27}$ requires an ionization of $1440 \mathrm{eV}$ ). The notable low signal at low charge states, $\mathrm{Xe}^{+}, \mathrm{Xe}^{+2}, \mathrm{Xe}^{+3}$ is believed to be due to the Auger relaxation processes that follow the initial photoionization, and results in the emission of 3 or 4 additional electrons.

The dominant signal in the spectra for xenon clusters (Figure 4 bottom) comes from low ionization charge states of xenon, from $\mathrm{Xe}^{+}$to $\mathrm{Xe}^{+6}$ (approximately $80 \%$ of the entire yield). Figure 4 shows the spectra for cluster of fixed size $(\langle N\rangle=11,000$ atoms) with varying LCLS pulse intensities spanning 2 orders of magnitude between $5.6 \times 10^{14} \mathrm{~W} / \mathrm{cm}^{2}$ and $4.5 \times 10^{16} \mathrm{~W} / \mathrm{cm}^{2}$. The abundance of low charge states at all these intensities can be understood from the formation of a Xe nanoplasma in the illuminated cluster which allows for three-body recombination during the hydrodynamic expansion of the plasma.

In the clusters, the photoionization and subsequent secondary ionization processes are still the main mechanisms for X-ray absorption and ionization. This is seen in the case of the the highest intensity $4.5 \times 10^{16} \mathrm{~W} / \mathrm{cm}^{2}$ when charge states of xenon up to $\mathrm{Xe}^{+26}$, similar to the atomic case, are obtained in a cluster environment. In the clusters, however, the photoelectrons and the Auger-electrons are trapped and will proceed to thermalize and heat the cluster. The outer layer is expelled rapidly in a Coulomb explosion, while the inner cluster core expands hydrodynamically on a longer time scale, allowing ion-electron recombination processes to take place. Measurements of the kinetic energy of Xe ions and electrons fit well with simulations of a hydrodynamic expansion of a plasma with an electron temperature of $125 \mathrm{eV}$, assuming an average ionization state of +5 per Xenon atom. ${ }^{26}$ 


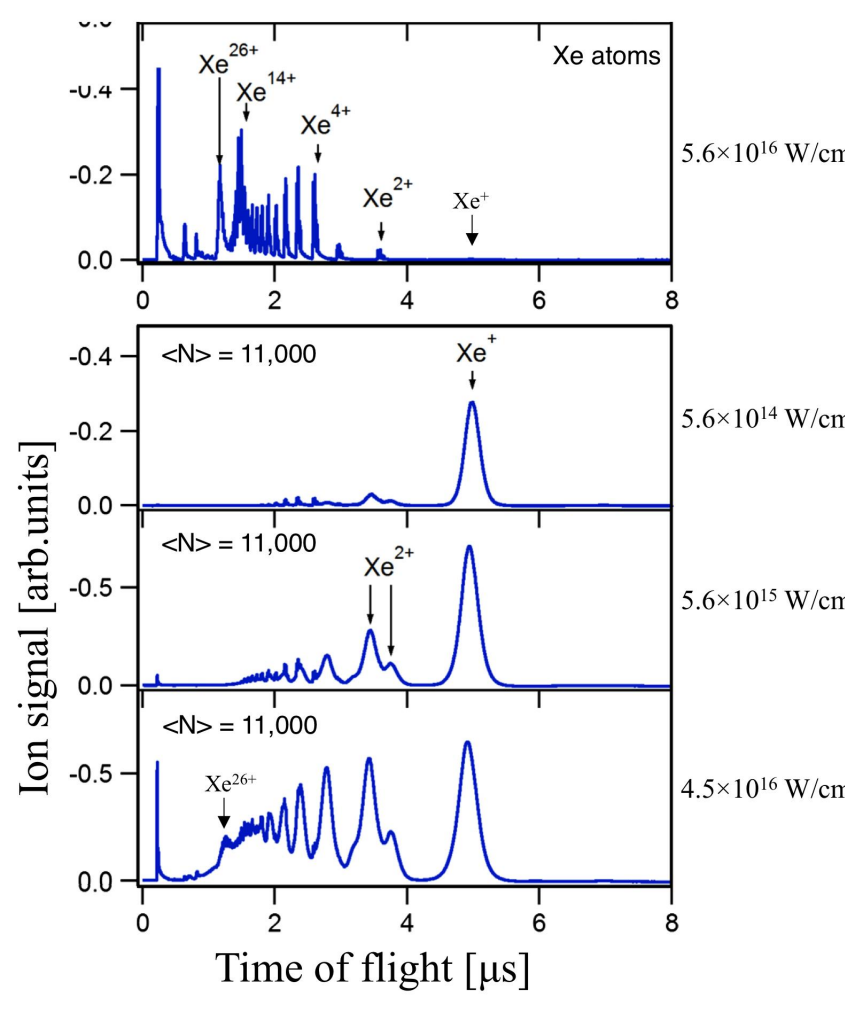

Figure 4. Time of flight spectra from Xenon atoms (top) and xenon clusters with a fixed size $\langle N\rangle=11,000$ (bottom), irradiated with single pulses at the LCLS. Pulse length $150 \mathrm{fs}$, photon energy $850 \mathrm{eV}$, varying pulse intensities. High charge states of xenon dominate the atomic spectrum reaching ionizations up to $\mathrm{Xe}^{+26}$. No $\mathrm{Xe}^{+}$is visible in the atomic spectrum, and appears only in the cluster spectra. Higher charge states are obtained in clusters at higher intensities. Figure adapted from. ${ }^{26}$

Calculations with a simple rate-equation model, ${ }^{34}$ which compute the charge states from the photoionization cross sections and Auger-emissions, collisional ionization and three-body recombination (between two low energetic electrons and one ion), describe quite well the observed charge states in the experiment. ${ }^{26}$ At the same time, our simulations with the non - local thermodynamics plasma code also agree with the experimental findings, predicting an electron temperature of $160 \mathrm{eV}$ (following a Maxwellian distribution) for the case of the highest intensity $5.6 \times 10^{16} \mathrm{~W} / \mathrm{cm}^{2}$ and electron densities above $10^{23} \mathrm{~cm}^{-3}$ in the plasma core. This supports further the proposed mechanism of three body recombination in the plasma environment following illumination.

\section{SUMMARY}

We have observed the explosion of large methane and xenon clusters irradiated by intense laser pulses in several experiments at FLASH $(92 \mathrm{eV})$ and LCLS $(850 \mathrm{eV})$. Fragmentation of these clusters proceeds by ejecting the outer charge layers, which is then followed by a hydrodynamic expansion driven by electron pressure. Such an environment facilitates recombination and leads to the production of molecular adducts or ion charge states which are not observed outside the cluster environment: $\mathrm{CH}_{5}$ molecules and higher adducts occur through molecular recombination and dominant $\mathrm{Xe}^{+}$charge states are the result of three-body recombination. These processes are dependent on the life-time of the expanding plasma core. 


\section{ACKNOWLEDGMENTS}

We thank Janos Hajdu for his support and fruitful discussions. Portions of this research were carried out at FLASH and LCLS, and we are grateful for access and support during the experiments. This work was supported by the following agencies: The Swedish Research Council, the Virtual Institute Program of the Helmholtz Association (VH-VI-302), the DFG Cluster of Excellence at the Munich Centre for Advanced Photonics, the German Research Foundation DFG through grant No. MO 719/6-1 and No. BO 3/69/2-2, the European Research Council, and the Knut and Alice Wallenberg Foundation, U.S. Department of Energy National Nuclear Security Administration under Cooperative agreement DE-FC52-03NA00156, the DOE Office of Basic Energy Sciences, the Welch foundation Grant no. F-1626. Access to FLASH was supported by the European Union under contract RII3-CT-2004-506008 (IA-SFS). The simulations were performed on resources provided by the Swedish National Infrastructure for Computing at UPPMAX, projects s00111-71 and s00112-67.

\section{REFERENCES}

[1] Feldhaus, J., "FLASH - the first soft x-ray free electron laser (FEL) user facility," J. Phys. B 43, 194002 (2010).

[2] Emma, P. et al., "First lasing and operation of an ngstrom-wavelength free-electron laser," Nature Phot. 4, $641-647(2010)$

[3] Young, L. et al., "Femtosecond electronic response of atoms to ultra-intense X-rays," Nature 466, 56 (2010).

[4] Wabnitz, H. et al., "Multiple ionization of atom clusters by intense soft X-rays from a free-electron laser," Nature 420, 482 (2002).

[5] Bostedt, C. et al., "Ultrafast X-Ray Scattering of Xenon Nanoparticles: Imaging Transient States of Matter," Phys. Rev. Lett. 108, 093401 (2012)

[6] Schorb, S. et al., "Size-Dependent Ultrafast Ionization Dynamics of Nanoscale Samples in Intense Femtosecond X-Ray Free-Electron-Laser Pulses," Phys. Rev. Lett. 108, 233401 (2012)

[7] Gorkhover, T. et al., "Nanoplasma Dynamics of Single Large Xenon Clusters Irradiated with Superintense X-Ray Pulses from the Linac Coherent Light Source Free-Electron Laser," Phys. Rev. Lett. 108, 245005 (2012)

[8] Chapman, H.N. et al., "Femtosecond time-delay X-ray holography," Nature 448, 676-679 (2007)

[9] Nagler, B. et al., "Turning solid aluminium transparent by intense soft X-ray photoionization," Nature Phys. $5,693(2009)$.

[10] Andreasson, J. et al., "Saturated ablation in metal hydrides and acceleration of protons and deuterons to keV energies with a soft X-ray laser," Phys. Rev. E 83, 016403 (2011).

[11] Chapman, H. N. et al., "Femtosecond X-ray protein nanocrystallography," Nature 470, 73-U81 (2011).

[12] Seibert, M.M. et al., "Single mimivirus particles intercepted and imaged with an X-ray laser," Nature 470, 78-U86 (2011).

[13] Boutet, S. et al., "High-Resolution Protein Structure Determination by Serial Femtosecond Crystallography," Science 337, 362-364 (2012)

[14] Redecke, L. et al., "Natively Inhibited Trypanosoma brucei Cathepsin B Structure Determined by Using an X-ray Laser " Science 339, 227-230 (2013)

[15] Neutze, R. et al., "Potential for biomolecular imaging with femtosecond X-ray pulses," Nature 406, 752 (2000).

[16] Last, I. et al., "Energetics and dynamics of Coulomb explosion of highly charged clusters ," J. Chem. Phys. $107,6685(1997)$

[17] Ditmire, T. et al., "Nuclear fusion from explosions of femtosecond laser-heated deuterium clusters," Nature 398, 489 (1999).

[18] Bostedt, C. et al., "Fast electrons from multi-electron dynamics in xenon clusters induced by inner-shell ionization," New J. Phys. 12, 083004 (2010)

[19] Bostedt, C. et al., "Multistep Ionization of Argon Clusters in Intense Femtosecond Extreme Ultraviolet Pulses," Phys. Rev. Lett. 100, 133401 (2008)

Proc. of SPIE Vol. 8777 87770J-7 
[20] Bergh, M. et al., "Model for the dynamics of a water cluster in an x-ray free electron laser beam," Phys. Rev. E 70, 051904 (2004)

[21] Hau-Riege, S. P. et al., "Dynamics of biological molecules irradiated by short x-ray pulses," Phys. Rev. E 69, 051906 (2004)

[22] Thomas, H. et al., "Shell explosion and core expansion of xenon clusters irradiated with intense femtosecond soft x-ray pulses," J. Phys. B: At. Mol. Opt. Phys. 42, 134018 (2009)

[23] Hoener, M. et al., "Charge recombination in soft x-ray laser produced nanoplasmas," J. Phys. B: At. Mol. Opt. Phys. 41, 181001 (2008)

[24] Ziaja, B. et al., "Emission of electrons from rare gas clusters after irradiation with intense VUV pulses of wavelength $100 \mathrm{~nm}$ and $32 \mathrm{~nm}, "$ New J. Phys. 11, 103012 (2009)

[25] Iwan, B., et al., "Explosion, ion acceleration, and molecular fragmentation of methane clusters in the pulsed beam of a free-electron laser," Phys. Rev. A 86, 033201 (2012)

[26] Thomas, H. et al., "Explosions of Xenon Clusters in Ultraintense Femtosecond X-Ray Pulses from the LCLS Free Electron Laser," Phys. Rev. Lett. 108, 133401 (2012)

[27] Tiedtke, K. et al., "The soft x-ray free-electron laser FLASH at DESY: beamlines, diagnostics and endstations," New J. Phys. 11, 023029 (2009).

[28] Hagena, O.F. and Obert, W.,"Cluster Formation in Expanding Supersonic Jets: Effect of Pressure, Temperature, Nozzle Size, and Test Gas," J. Chem. Phys. 56, 1793 (1972)

[29] Madison, K. W., et al., "Fusion neutron and ion emission from deuterium and deuterated methane cluster plasmas ," Phys. Plasmas 11, 270 (2004)

[30] Scuseria G.E., "The elusive signature of $\mathrm{CH}_{5}^{+}$," Nature 366, 512 - 513 (1993)

[31] Scott, H. A., "Cretin - a radiative transfer capability for laboratory plasmas," J. Quant. Spectrosc. Radiat. Transfer 71, 689 (2001).

[32] Scott, H. A. et al., "Advances in NLTE modeling for integrated simulations," High Energy Density Physics 6, 39 (2009).

[33] Hau-Riege, S. P. et al., "Sacrificial Tamper Slows Down Sample Explosion in FLASH Diffraction Experiments," Phys. Rev. Lett. 104, 064801 (2010)

[34] Ditmire, T. et al., "Interaction of intense laser pulses with atomic clusters," Phys. Rev. A 53, 3379-3402 (1996) 